\title{
New way for the innovation of gear types
}

\author{
László Dudás \\ University of Miskolc \\ Hungary
}

\section{Introduction}

Gears are one of the most complicated parts of machines. Their efficiency and optimum design determines the success of the machine. The first level of innovation is the geometry. The geometry determines the possible usage area of the gearing and influences the manufacturability and working parameters of the gear. Consequently, improvement and innovation of gear geometry is always a focus of interest for gear manufacturers. For these reasons, many gear-oriented CAD software packages have been developed. Figure 1 gives a comparison of several programs depending on their suitability for dimensioning or development. The design tool ZPS (Miltenovic \& Milcic, 1999) or KISSsoft (Kissling, 1999) is mainly intended for the determination of suitable sizes for a given gearing type to fulfil the construction requirements. The programs ZAKGEAR (Lunin, 2010), UMCORR (Stadtfeld, 1999), KEGELRADKETTE (Landvogt et al., 1999) or Kato's application (Kato \& Kubo, 1999) are more suitable for the development of new characteristics of gearings or for optimisation.

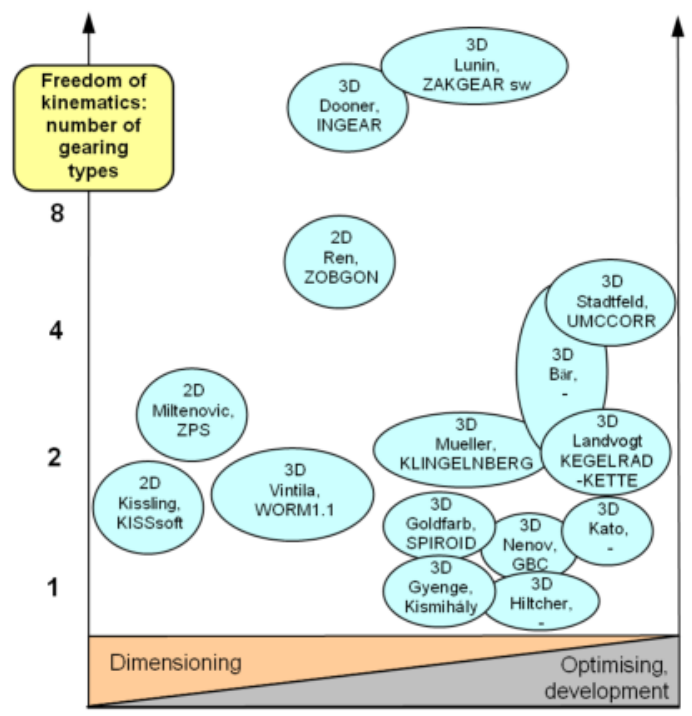

Fig. 1. Comparison of gearing design programs 
The vertical axis measures the number of different modelled gear types. One of the programs having the largest freedom of embedded gearing kinematics is ZAKGEAR sw (Lunin, 2010). Most of the programs are intended to handle a few concrete gear types and the number of modelled sorts is limited. Recognition of this fact motivated the author to find a robust connection theory and to create a surface and kinematical relation independent gearing development tool. The name of this connection theory is the "Reaching Model". The original theory will be introduced with full particulars here. This theory serves as the basis for the developed Surface Constructor kinematical modelling and simulation tool, the goal of which is to provide maximum freedom in modelling contacting kinematical surfaces and their kinematical arrangements. The tool's capabilities will be demonstrated through modelling of innovative gear constructions.

\section{The theory}

To give a basis for referencing in the modelling section, we need to go over the theoretical fundamentals of the innovative Surface Constructor kinematical surface generating and contact analysing software. The name of the theory introduced by the author for generation conjugate surface pairs is the Reaching Model. Here we give an explanation with emphasis on its novelty and differences from other models. The model solves the well-known task of gearings - the determination of the $F 2$ conjugate surface if the generating $F 1$ surface and the generating motion are given. Figure 2 shows the two surfaces with their holder frames K1 and $K 2$. The $\kappa$ curved co-ordinate system plays a major role in the Reaching Model theory.

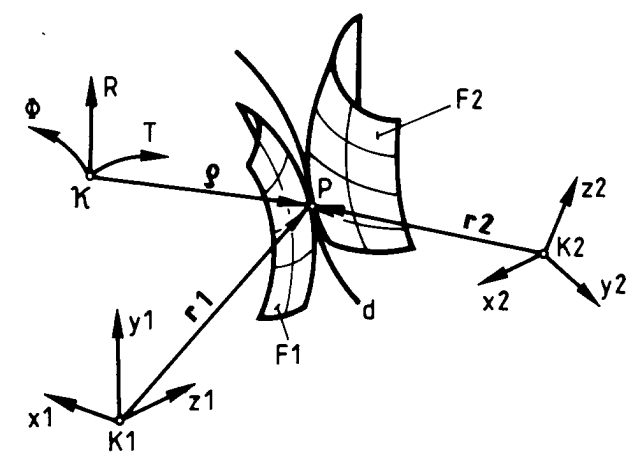

Fig. 2. Generating surface F2 by surface F1

There are two well-known methods to solve this task:

- the differential-geometric method developed by Gochman, and

- the kinematical method, which applies the $\boldsymbol{n} \cdot \boldsymbol{v}^{1,2}=0$ scalar product where $\boldsymbol{n}$ is the normal vector and $\boldsymbol{v}^{1,2}$ is the relative speed vector at the contacting point. This method has two forms: the pure geometrical and the Litvin type, which uses matrix-algebra (Litvin, 1994). 


\subsection{The Reaching Model}

Before the Reaching Model the word undercut was used for various undesirable surface problems of mating surface pairs. The Reaching Model distinguishes local undercut situations from global cut. The methods mentioned above to determine the unknown surface are local methods, i.e., they can produce the theoretical mating points and the border of areas containing such points. The border points are points where local undercut occurs. The local undercut, which can appear in an infinitely small space and time domain, is practically always accompanied by global cut, when one part of the local area is destroyed by the other surface, e.g., the enveloped surface of the tooth of a worm-wheel is damaged by the tip edge of the worm. Though global cut usually follows local undercut, it can appear alone, as well. In our terminology the word interference may mean either local undercut or global cut. To determine local undercut locations infinitesimal calculation is needed. To determine the location of global cut usually iterative or discrete simulation methods are used. One method for discovering interferences is the use of normal, then inverse generating processes comparing the result with the original surface. Another solution (Seveleva et al., 1989) applied two theoretically different methods - in our terminology a local and a global method - to generate the needed surface and if these surfaces were identical then there was no interference.

The Reaching Model includes the capability to detect all types of local undercuts and the global cut in the same theoretical model. The main advantage of the model is its simplicity. In this model the generation of one of the points of the F2 surface is equal to solving a simple minimum value problem. The denominative reaching process will be introduced briefly with the help of Figure 3.

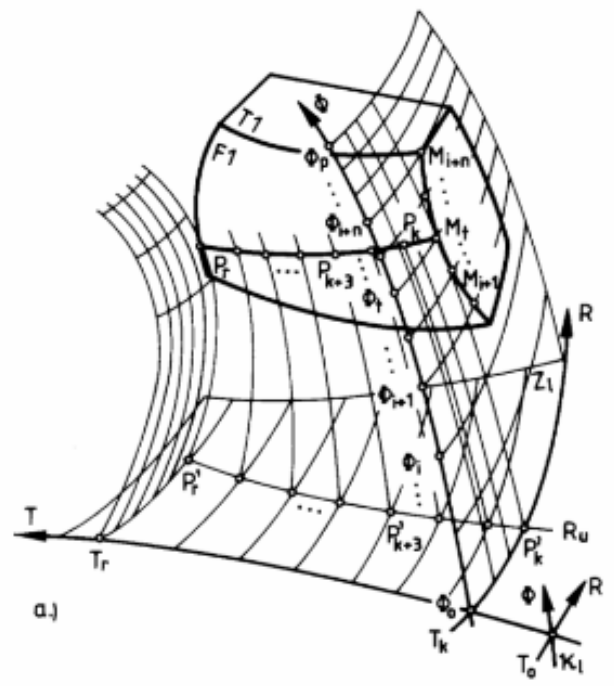

Fig. 3. The reaching process

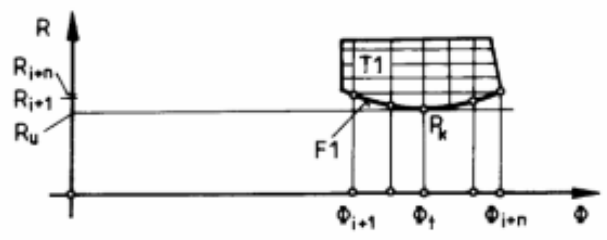

b.) 
The Reaching Model applies a special non-Descartes co-ordinate system $\kappa$. The $\Phi$ coordinate lines as well as the co-ordinate axis $\Phi$ itself coincide with the motion paths of the points of a surface that is in the co-ordinate system $K 2$, meaning that $\Phi$ has two roles:

1. motion (time) parameter,

2. one of the three space co-ordinates of co-ordinate system $\kappa$.

In the reaching process we choose a $\Phi$ co-ordinate line that does not intersect the $T 1$ body. Stepping from one $\Phi$ co-ordinate line to another going in $R$ direction, the generating $\Phi$ coordinate line will be that which can reach $F 1$ first. This $\Phi$ line is the path of motion of point $P_{k}$ ' that will be one surface point of the generated surface F2. Point $P_{k}$ will be the contact point, see Figure 3a. Transforming the intersection of the $R-\Phi$ co-ordinate surface to a Descartes co-ordinate system, it is evident that the determination of a $P_{k}$ point is equal to solve a minimum-value problem, as shown in Figure $3 \mathrm{~b}$.

The necessary condition of connection in the Reaching Model is

$$
\frac{\partial R}{\partial \Phi}=0
$$

where $R=R(\Phi, T, Z)$ is the reaching-coordinate function, $\Phi$ is the motion-path co-ordinate, $T$ is the division co-ordinate in the $\kappa$ slicing co-ordinate system, $Z$ is the identifying parameter of the $\kappa$ co-ordinate system. This necessary condition is equivalent to the $n \cdot v^{1,2}=0$ condition of the above-mentioned kinematical method.

The sufficient condition of the local minimum in the $\Phi=\Phi_{t}$ point that is equivalent to the real connection at the same location is given in the following form:

$$
\begin{aligned}
& \left.\frac{\partial^{v} R}{\partial \Phi^{v}}\right|_{\Phi=\Phi_{t}}=0 \quad(v=1,2, \ldots, w-1) \text { and } \\
& \left.\frac{\partial^{w} R}{\partial \Phi^{w}}\right|_{\Phi=\Phi_{t}}>0 \quad \text { where } w \text { is an even number. }
\end{aligned}
$$

This condition defines a local minimum point at $\Phi=\Phi_{t}$ which generates the $P_{k}{ }^{\prime}=P_{k}{ }^{\prime}\left(\Phi=\Phi_{t}\right.$; $R=R\left(\Phi_{t}\right) ; T=T_{k}$ ) point of the calculated $F 2$ surface. The local nature of this condition means that it can be fulfilled in an infinitesimally small time or space region, but a generating process with a longer $\Phi$ (time and space) interval may destroy the generated $P_{k}{ }^{\prime}$ point.

\subsection{Situations of local undercut}

The model can give all the types of local undercuts simply by discussing the minimum value problem in a local tangential point. To recognize different types of local undercuts we need the path of motion of point $P_{k}{ }^{\prime}$ only. If the necessary local condition of contact becomes true and the path of motion intersects the solid T1 there will be local undercut.

\section{- First Situation}

As shown in the left subfigure of Figure 4, $\Phi_{t}$ is a local maximum point of $R=R(\Phi)$ function. The path of motion of point $P_{k}^{\prime}$ interferes with solid T1. 

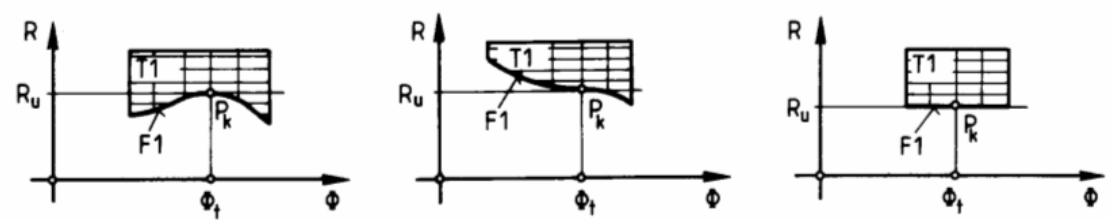

Fig. 4. Situations of local undercut

The sufficient local condition of undercut is as follows:

$$
\begin{aligned}
& \left.\frac{\partial^{v} R}{\partial \Phi^{v}}\right|_{\Phi=\Phi_{t}}=0 \quad(v=1,2, \ldots, w-1) \text { and } \\
& \left.\frac{\partial^{w} R}{\partial \Phi^{w}}\right|_{\Phi=\Phi_{t}}<0 \quad \text { where } w \text { is an even number. }
\end{aligned}
$$

- Second Situation

The general type of local undercut is shown in the middle subfigure of Figure 4 . In this situation an inflexion form yields undercut. The sufficient local condition of this type of undercut is given as:

$$
\begin{aligned}
& \left.\frac{\partial^{v} R}{\partial \Phi^{v}}\right|_{\Phi=\Phi_{t}}=0 \quad(v=1,2, \ldots, w-1) \text { and } \\
& \left.\frac{\partial^{w} R}{\partial \Phi^{w}}\right|_{\Phi=\Phi_{t}} \neq 0 \quad \text { where } w \text { is an odd number. }
\end{aligned}
$$

This situation includes a more complex form, shown in Figure 5.

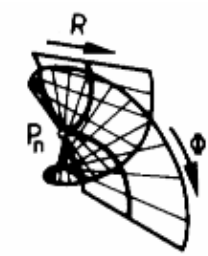

a.)

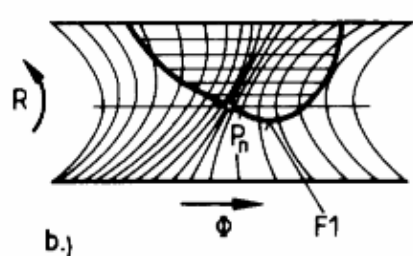

b.)

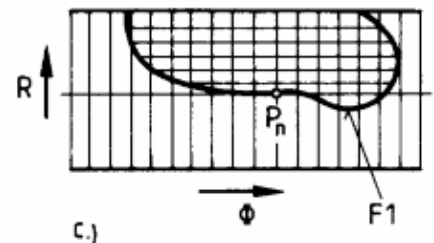

c.)

Fig. 5. The motion of point $P_{k}{ }^{\prime}$ stops at point $P_{n}$ for a moment

The motion of the point $P_{k}{ }^{\prime}$ comes to a halt for a moment (the speed of point $P_{k}{ }^{\prime}$ becomes 0 for a moment). Figure 5 demonstrates this case in three dimensions (a), after one step of 
transformation (b) and at the end of transformation from the curved $\Phi-R$ coordinate surface to the coordinate system that has straightened $\Phi-R$ coordinate lines (c). This special case is equivalent to the inflexion situation shown in Figure 4 and it can be detected using the same sufficiency condition.

- Third Situation

If the $R=R(\Phi)$ function of the intersection of surface $F 1$ is constant in the infinitely small surroundings of point $P_{k}$ as represented in the right subfigure of Figure 4, the sufficient condition of this type of local undercut shows the following form:

$$
\left.\frac{\partial^{w} R}{\partial \Phi^{w}}\right|_{\Phi=\Phi_{t}}=0 \quad \text { where } w=\infty \text {, infinite value. }
$$

In this third situation the speed of the contact position on F2 becomes zero because the same $P_{k}^{\prime}$ generated point connects to $F 1$ for a short time. This type of local undercut may be theoretical if the generated surface $F 2$ is smooth in this $P_{k}{ }^{\prime}$ contact point. Though this does not create an edge on the generated F2 surface, the momentary or prolonged lack of motion results in decreased pressure in the oil film and is consequently undesirable.

Sometimes an enveloped edge is formed on the surface F2, like on the gear of a Cone-type classical globoid gearing. This case can be analysed in Figure 6. One of the paths of motion coincides with intersection of generating surface $F 1$, as shown in Figure 6a. In this general case the angle $\beta$ between the path of motion and coordinate direction $R$ changes point by point. Figure $6 \mathrm{~b}$ shows the same example but the generated surface $F 2$ is fixed and the generating solid $T 1$ (and its surface F1) is moving. The result is an enveloped edge on surface $F 2$, and point $P_{k}{ }^{\prime}$ of $F 2$ remains in continuous contact with surface $F 1$ for a certain time. Figure $6 \mathrm{c}$ demonstrates the function $\beta=\beta(\Phi)$ in this general case. If the function $\beta$ is not constant then an edge will appear on the generated surface F2; otherwise the surface will be smooth.

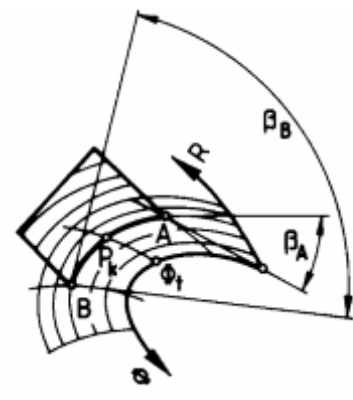

a.)

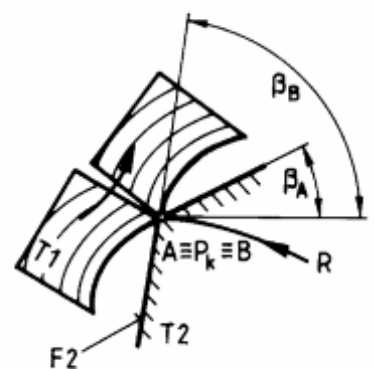

b.)

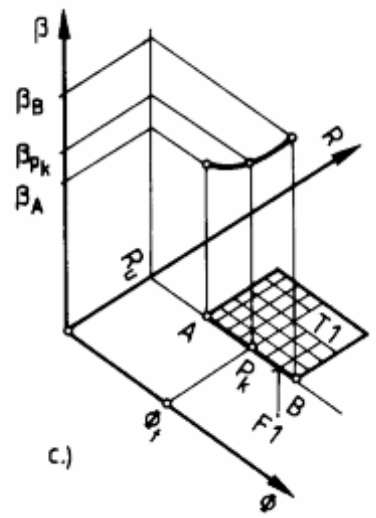

c.) 


\subsection{The cut as a global phenomenon}

As shown in Figure 7, the path of generated point $P_{k}$ interferes with the solid $T 1$ in the represented space and corresponding time interval. The $P_{k}{ }^{\prime}$ point of the $F 2$ surface generated by the $P_{k}$ local minimum point at $\Phi_{t}$ value will be destroyed by a cut if the motion interval is not limited.

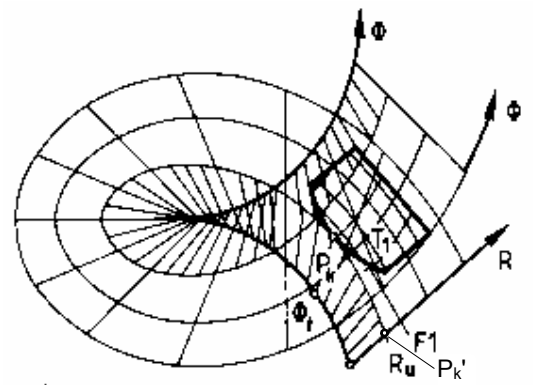

a.)

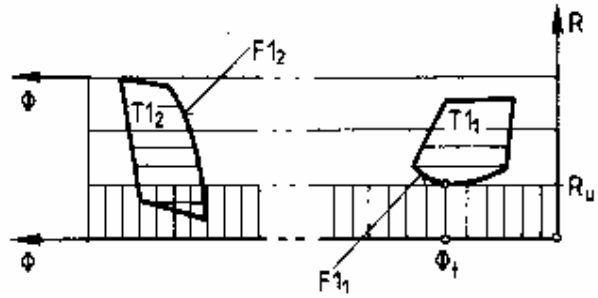

b.)

Fig. 7. An example of global cut

\section{The application}

Though the theory works with analytical expressions and partial derivatives, a robust, surface-independent software for realisation of the theory was developed on a discrete numerical basis. This program is named Surface Constructor (SC) and will be demonstrated in the following sections. The developed system applies both symbolic and numerical representations of the objects. The symbolic algebraic computation provides the flexible characteristic of the tool. SC starts as an empty kinematical modelling shell and models the kinematical modelling process itself. The system sketched in Figure 8 has three main representation levels:

- the symbolic level, which uses a symbolic algebraic representation of the objects in the kinematical model,

- $\quad$ the numerical level, which stores the given and computed objects using numerical form, and

- the visualisation level, which allows the analysis of views and motion of the objects.

The selection and visualisation options are as follows:

- F2glob: global computational method and appropriate result

- F2lok: local computational method and appropriate result

- F2al: computation and visualisation of the occurrences of local undercuts

- $\Phi$ : computation and visualisation of moving path of selected points

- $\quad R-\Phi$ : computation and visualisation of $R=R(\Phi)$ functions as a special feature of this software

- $\quad v_{a} a$ : computation and visualisation of the space of relative speed and acceleration

- $\quad P T$ : computation and visualisation of axoids. 


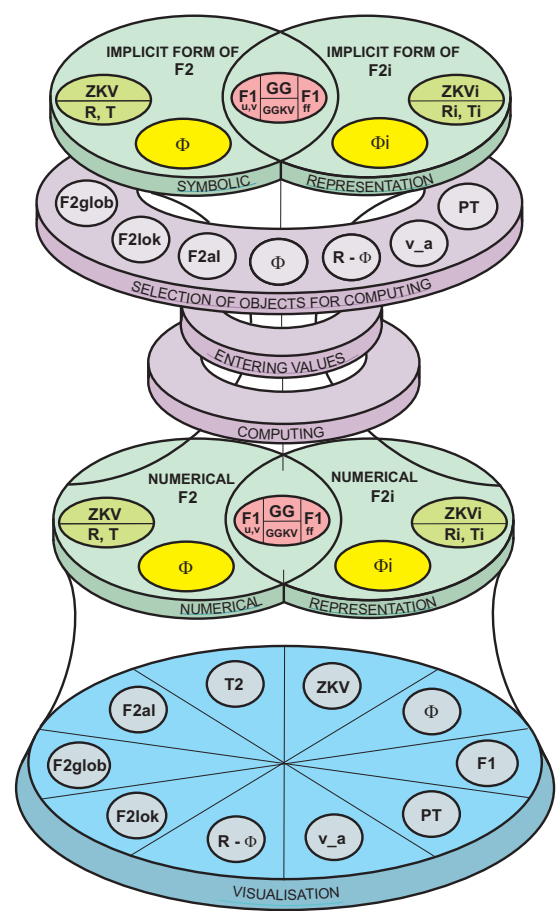

Fig. 8. The structure of the Surface Constructor kinematical modelling tool

The application can envelope different $F 2 i$ surfaces by the same generating $F 1$ surface, modelling point-like connections in such a way. In the case of $i=2$ the generated members of a gearing with a point-like connection are surfaces $F 2_{1}$ and $F 2_{2}$. The intermediary theoretical generating surface is F1. The generating kinematical relations are given by transformation matrices as functions of Fi1 and Fi2 motion parameters. In the software and in the followings Fi will replace $\Phi$. Similarly, the Rho, Tau and Zeta coordinates correspond to the $R, T$ and $Z$ coordinates of the described theory. Moreover SC changes each letter to upper case form.

The usability of the computer program for modelling of gear members can be followed in the next figures. Figure 9 shows the results of modelling a ZTA-type worm gearing. The result of global cut produced by the top edge of the hob can be detected on the right side of the calculated teeth. Comparing it to the teeth of the real worm-wheel proves that the program simulates the real cutting conditions well.

\subsection{Visualization of $R=R(\Phi)$ functions}

Most modern programs apply the TCA (Tooth Contact Analysis) method for optimizing contacting properties (Su \& Qin, 2003; Litvin \& Fuentes, 2004). One of the best realizations uses real time ease-off topography manipulation through surface or kinematical system parameter modification using mouse and visual feedback (Stadtfeld, 1999). But this and similar methods need the two mating surfaces previously computed in the case of conjugate contacting too. 

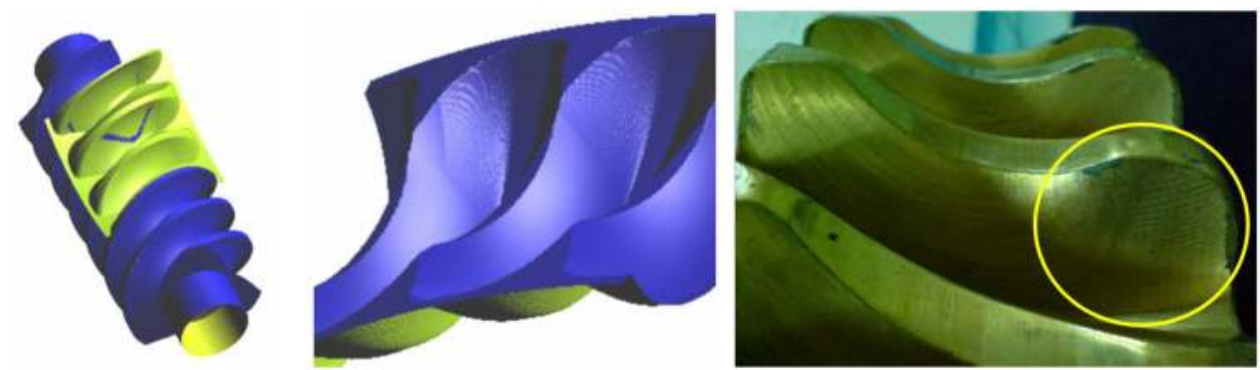

Fig. 9. The ZTA worm gearing with the contact line, the calculated worm wheel segment and the manufactured real worm wheel surface

The investigation in the space of path of motion characterizes SC only. Using this new tool, we can optimize contacting characteristics of connection and avoid interference situations without the generated F2 surface. From the description given above it is evident that the curves of $R=R(\Phi)$ functions are very suitable for detecting different types of local undercuts and global cuts of contacting surfaces. These occurrences are very dangerous not only in gearing contact but in cutting gearing parts, too. The analyzing of $R=R(\Phi)$ functions offers a unique possibility, since by using this facility it is possible to avoid these problems. Good contact is characterized by a global minimum point, whereas problematic local undercut situations are featured by points having inflection with horizontal tangent, or local maximum. Figure 10 shows examples.

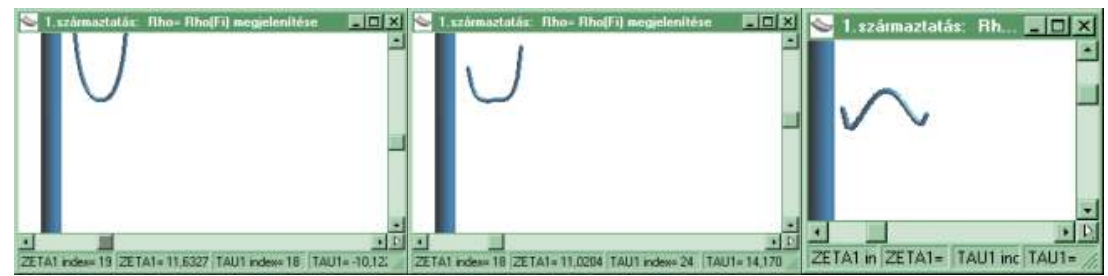

Fig. 10. The investigation window for selecting the needed $R=R(\Phi)$ function or set of functions using mouse

Curves having a local maximum point often produce global cut that is the horizontal tangent of local maximum point intersects the curve in a farther point. One $R=R(\Phi)$ curve characterizes the generating process of a point of the $F 2$ generated surface. If we would like to analyze all the grid-points of the generated $F 2$ surface we have to study a set of $R=$ $R(\Phi, T)$ surfaces. This can be carried out conveniently using the input interface shown in Figure 11. To provide the maximum comfort the input interface is the visualization window itself.

Using the mouse, the following visualization possibilities are given:

- A click on the $M$ grid-point results in the visualization of an $R=R(\Phi)$ function which characterizes the generating process of the $P 2(T, Z)$ point of the $F 2$ generated surface

- Dragging the $M$ point results in a continuously changing $R=R(\Phi)$ function curve

- A click on the $T$ or $Z$ adjuster results in visualization of an $R=R(\Phi, Z)$ or $R=R(\Phi, T)$ surface 


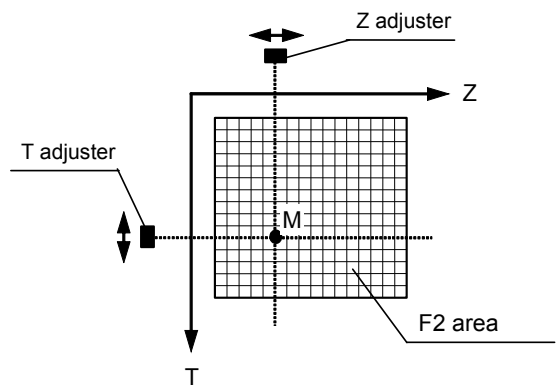

Fig. 11. The adjusting area for selecting the needed $R=R(\Phi)$ function associated to $M$ point

- Dragging the $T$ or $Z$ adjuster gives a continuously changing $R=R(\Phi, Z)$ or $R=R(\Phi, T)$ surface. The form of the surface characterizes the quality of contact between the given $F 1$ surface and the points of one line of the needed F2 surface. Good contact between F1 and F2 will occur if every $R=R(\Phi, \mathrm{Z})$ or $R=R(\Phi, T)$ surface has a 'valley'. The visualization of surfaces is possible applying a set of $R=R(\Phi)$ curves or drawing a rendered surface, as shown in Figure 12.

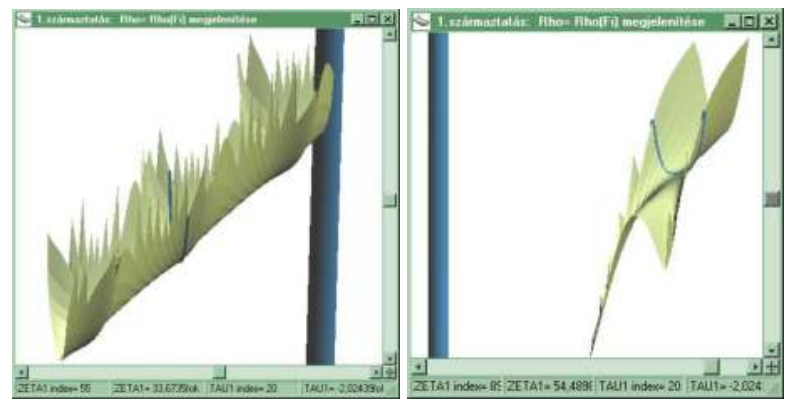

Fig. 12. To the left: Good conjugate contact along the ZETA1 = constant parameter curve of $F 2$. To the right: The $R=R(\Phi)$ curve indicates the appearance of the undercut point on $F 2$.

\subsection{Optimizing the contacting properties applying visual control of changing $R=R(\Phi)$ curves, surfaces}

This optimizing capability characterizes only this design tool. In most cases the F1 given surface is changeable, or the kinematical relations have some flexibility. In these situations we can optimize the contacting properties by changing the value of one of the parameters of F1 surface or the kinematical system. If we have a powerful enough computer system this changing of the selected value may be carried out in a continuous manner using visual control of $R=R(\Phi)$ curves or surfaces. The result will be similar to a situation when the parameter change is resulted by direct manipulation of the surface using mouse dragging.

To realize this possibility we have to apply a comfortable user interface for changing parameter values, for example sliders. If there is any deviant pattern on the selected $R=$ $R(\Phi)$ curve or surface it is possible to make it disappear by manipulating the parameter by its slider. 


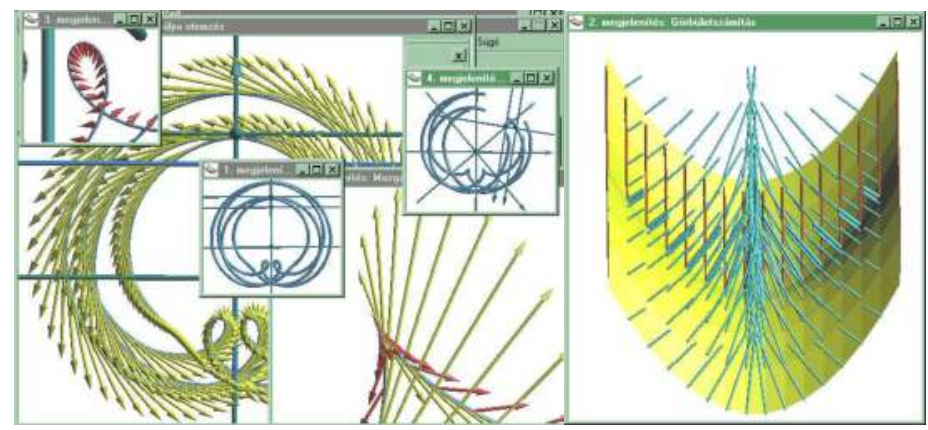

Fig. 13. Displaying space properties and curvature values in different windows

\subsection{Visualization of space properties}

The features of the motion can be visualised drawing the path of motion of given points, the speed and the acceleration vectors, the curvature values. Figure 13 demonstrates the possibilities.

\subsection{Visualization of instantaneous screw axis and axoid}

One of the unique capabilities of SC is the real-time visualization of an instantaneous screw axis, and the set of axes as axoid. The axoids of simple relative motions are known as surfaces of revolution, e.g. cylinders or cones. The goal of SC is to visualise axoids in more complicated motion as well. This section will describe the mathematical and geometrical basics of calculating an instantaneous screw axis if given the space of speed of the relative motion. The screw axis may be defined between two connected co-ordinate systems moving relative to each other. In a real situation these frames hold gears that may be considered as rigid solids. The space of relative speed in every moment forms a screw, the speed vectors are tangential to spirals, to helical curves. The main task is to determine the position of the axis of this screw in the space. See Figure 14 for an example. In case of continuous motion this process will result in a set of axes that form a line-surface, the axoid. This axoid may be shown relative to a selected member of the kinematical model and these surfaces have different shapes in general.

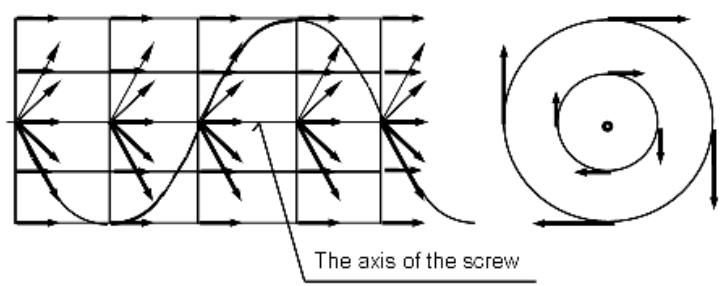

Fig. 14. The speed vectors are tangential to helical curves in a general screw

In the following section the calculation of the position of the momentary turning axis will be presented. The calculation introduced here differs from the discussion of pitch surfaces and velocity screws given in (Ramahi, 1998), because it uses the velocity vectors of the screw 
directly to determine the screw axis. This method does not need angular velocity or gear ratio, etc. It needs only three - properly selected - velocity vectors from the space, from the screw. In this calculation the $K 1$ and $K 2$ co-ordinate systems move relative to each other and suppose that $K 2$ is fixed and $K 1$ is moving. The first step determines the direction of the axis in the $K 2$ frame while second gives one of the points of the axis.

Determining the direction of the screw axis

To determine the direction of the axis we have to take into consideration the fact that the component parallel to the axis of every speed vector is equal. Look again at the screw in Figure 14. From here, we select three speed vectors and translate them to the origin of the K2 co-ordinate system as shown in Figure 15.

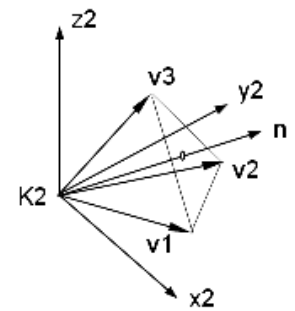

Fig. 15. The three velocity vectors are selected randomly from the screw

The three velocity vectors form a pyramid. The height line will give the direction of the axis of the screw. In Figure 15 it is marked as the normal vector of the base triangle of the pyramid. Equation (9) gives the $n$ normal in mathematical form.

$$
n=\left(v_{2}-v_{1}\right) \times\left(v_{3}-v_{2}\right)
$$

The normalised vector is then:

$$
n_{0}=n /|n|
$$

Determining one of the points of the screw axis

To determine one of the points of the screw axis, we need to transform the three velocity vectors and their start points from the K2 co-ordinate system to a $K 2 "$ system. The $z^{\prime \prime}$ axis of this K2" system is parallel to the $n$ normal vector, so the projection of the three velocity vectors will be tangential to concentric circles on the $x^{\prime \prime}-y^{\prime \prime}$ co-ordinate plane. The coordinate transformation is shown in Figure 16.

The transformation matrix consists of two consecutive rotations. The first rotation matrix rotates by $\alpha$ :

$$
M_{2,2}=\left[\begin{array}{cccc}
1 & 0 & 0 & 0 \\
0 & \cos \alpha & -\sin \alpha & 0 \\
0 & \sin \alpha & \cos \alpha & 0 \\
0 & 0 & 0 & 1
\end{array}\right]
$$




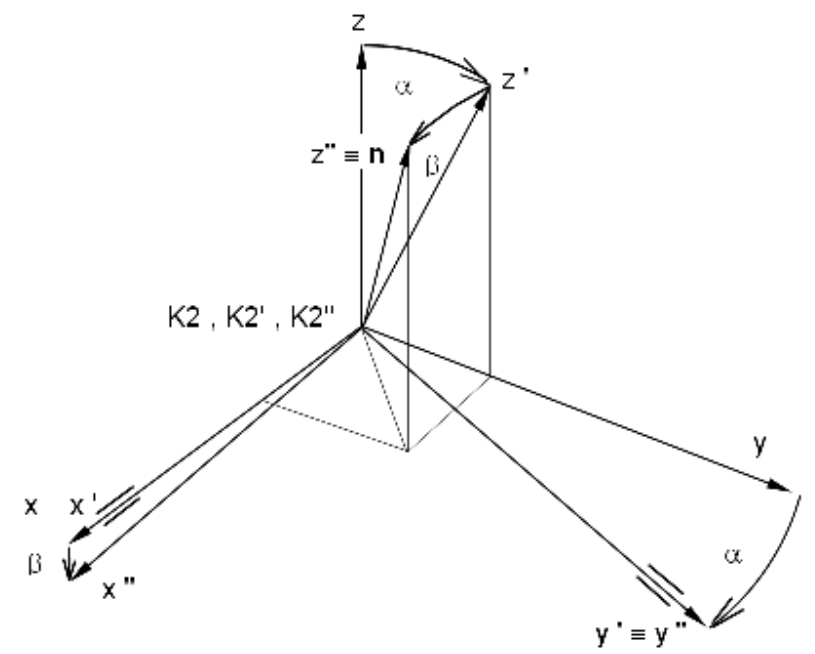

Fig. 16. In the K2" co-ordinate system the $z^{\prime \prime}$ co-ordinate axis is parallel to $n$

The second rotation matrix rotates by $\beta$ :

$$
M_{2^{\prime \prime}, 2^{\prime}}=\left[\begin{array}{cccc}
\cos \beta & 0 & \sin \beta & 0 \\
0 & 1 & 0 & 0 \\
-\sin \beta & 0 & \cos \beta & 0 \\
0 & 0 & 0 & 1
\end{array}\right]
$$

The resultant transformation matrix is:

$$
M_{2^{\prime \prime}, 2}=M_{2^{\prime \prime}, 2^{\prime}} \cdot M_{2^{\prime}, 2}
$$

The values for $\alpha, \beta, \sin \alpha, \cos \alpha, \sin \beta$ and $\cos \beta$ from Figure 16 are:

$$
\begin{gathered}
\alpha=\operatorname{arctg}\left(n_{y} / n_{z}\right), \quad \sin \alpha=n_{y} / \sqrt{n_{y}^{2}+n_{z}^{2}}, \quad \cos \alpha=n_{z} / \sqrt{n_{y}^{2}+n_{z}^{2}} \\
\beta=\operatorname{arctg}\left(n_{x} / \sqrt{n_{y}^{2}+n_{z}^{2}}\right), \quad \sin \beta=n_{x} / \sqrt{n_{x}^{2}+n_{y}^{2}+n_{z}^{2}}=n_{0 x}, \\
\quad \cos \beta=\sqrt{n_{y}^{2}+n_{z}^{2}} / \sqrt{n_{x}^{2}+n_{y}^{2}+n_{z}^{2}}=\sqrt{n_{0 y}^{2}+n_{0 z}^{2}}
\end{gathered}
$$

To transform a $P_{i}$ point given by $r_{i}$ from the $K 2$ frame to the $K 2^{\prime \prime}$ frame, the $M_{2 ", 2}$ is needed:

$$
r_{i}^{\prime \prime}=M_{2 ", 2} \cdot r_{i}
$$


Determining one of the points of the screw axis is equal to determining its intersection point in the $z^{\prime \prime}=0$ co-ordinate plane. This task will be carried out in this plane, so only the $x^{\prime \prime}$ and $y^{\prime \prime}$ components of the vectors are needed. The projections of the velocity vectors on the $z^{\prime \prime}=$ 0 plane are tangential to concentric circles, so the perpendicular lines intersect each other at the desired Pt point as shown in Figure 17.

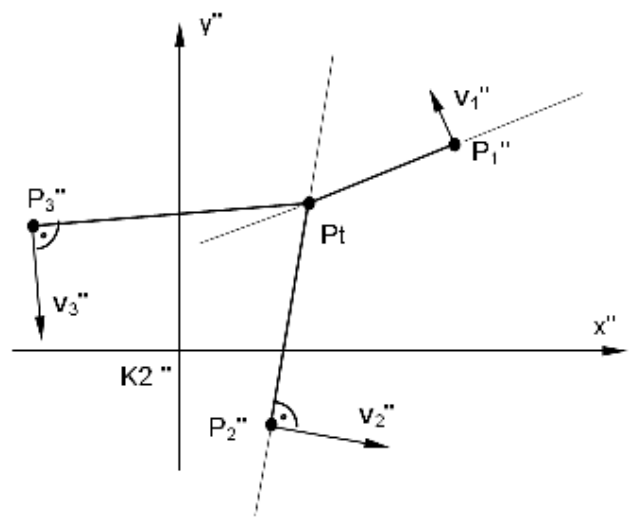

Fig. 17. Determining the $P t$ intersection point as a point of the screw axis

If the projected velocity vectors are $v_{1}^{\prime \prime}=\left(x_{v 1} ; y_{v 1}\right) ; v_{2}^{\prime \prime}=\left(x_{v 2} ; y_{v 2}\right)$, then the perpendicular normal vectors are the following: $n_{v 1}=\left(y_{v 1} ;-x_{v 1}\right) ; n_{v 2}=\left(y_{v 2} ;-x_{v 2}\right)$.

The intersection condition is:

$$
r_{1}{ }^{\prime}+k 1 \cdot n_{v 1}=r_{2}^{\prime \prime}+k 2 \cdot n_{v 2}
$$

In scalar form:

$$
\begin{aligned}
& x_{r 1}^{\prime \prime}+k 1 \cdot y_{v 1}=x_{r 2}^{\prime \prime}+k 2 \cdot y_{v 2} \\
& y_{r 1}^{\prime \prime}-k 1 \cdot x_{v 1}=y_{r 2}^{\prime \prime}-k 2 \cdot x_{v 2}
\end{aligned}
$$

from which:

$$
\begin{aligned}
& k 1=\left(y_{r 2}^{\prime \prime} \cdot y_{v 2}-y_{r 1}^{\prime \prime} \cdot y_{v 2}+x_{r 2}^{\prime \prime} \cdot x_{v 2}-x_{r 1}^{\prime \prime} \cdot x_{v 2}\right) /\left(x_{v 2} \cdot y_{v 1}-x_{v 1} \cdot y_{v 2}\right) \\
& k 2=\left(y_{r 2}^{\prime \prime} \cdot y_{v 2}-y_{r 1}^{\prime \prime} \cdot y_{v 2}+x_{r 2}^{\prime \prime} \cdot x_{v 2}-x_{r 1}^{\prime \prime} \cdot x_{v 2}\right) /\left(x_{v 2} \cdot y_{v 1}-x_{v 1} \cdot y_{v 2}\right)
\end{aligned}
$$

The $k 1$ and $k 2$ values have to be identical.

The co-ordinates of wanted Pt intersection point in the K2" frame are: 


$$
\begin{aligned}
& x_{P t}^{\prime \prime}=x_{r 1}^{\prime \prime}+k 1 \cdot y_{v 1} \\
& y_{P t}^{\prime \prime}=y_{r 1}^{\prime \prime}-k 1 \cdot x_{v 1} \\
& z_{P t}^{\prime \prime}=0
\end{aligned}
$$

The $P t$ intersection point in the $K 2$ frame may be calculated using an inverse transformation:

$$
r_{P t}=M_{2,2^{\prime \prime}} \cdot r_{P t}^{\prime \prime}
$$

where $\quad M_{2,2^{n}}=M_{2^{\prime \prime}, 2}^{-1}$, but now $M_{2,2^{\prime \prime}}=M_{2^{\prime \prime}, 2}^{T}$ because there was only rotation.

$$
M_{2,2 \text { " }}=\left[\begin{array}{cccc}
\cos \beta & 0 & -\sin \beta & 0 \\
\sin \alpha \cdot \sin \beta & \cos \alpha & \sin \alpha \cdot \cos \beta & 0 \\
\cos \alpha \cdot \sin \beta & -\sin \alpha & \cos \alpha \cdot \cos \beta & 0 \\
0 & 0 & 0 & 1
\end{array}\right]
$$

The line of the momentary axis

Finally the axis line of the momentary velocity screw can be given in the $K 2$ frame using the $p$ parameter:

$$
r=r_{P t}+p \cdot n_{0}
$$

The velocity vector of the momentary sliding:

$$
v_{2,1}=\left(n_{0} \cdot v_{1}\right) \cdot n_{0}
$$

The angle speed of the momentary rotation:

$$
\varpi=m 1 / \sqrt{\left(x_{r 1}^{\prime \prime}-x_{P t}^{\prime \prime}\right)^{2}+\left(y_{r 1}^{\prime \prime}-y_{P t}^{\prime \prime}\right)^{2}}
$$

The parameter of the momentary screw:

$$
P=\left|v_{2,1}\right| / \varpi
$$

and the pitch:

$$
P_{a x}=2 \cdot \pi \cdot P
$$

This calculation is based on the velocity space itself and fits well to its embedding software environment. The visualisation of the set of axes as axoid helps gearing developers to study the character of kinematical relations. Figure 18 shows the axoid of a bevel gearing and the case of a gearing having out-of-line axes. Figure 19 shows the transformation matrix and the axoid of a complex motion. 


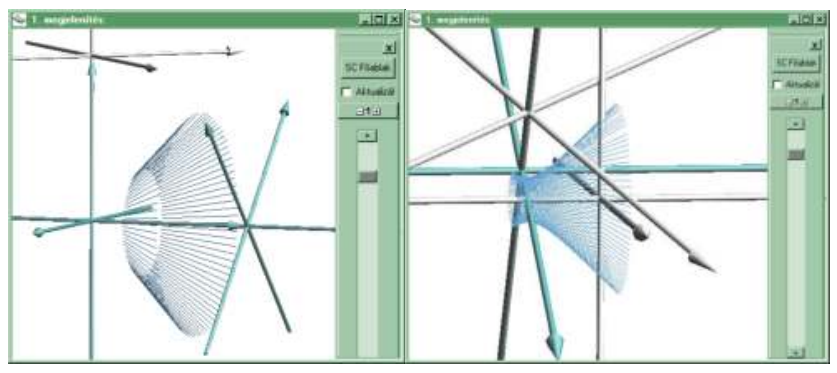

Fig. 18. Axoids of simple motions
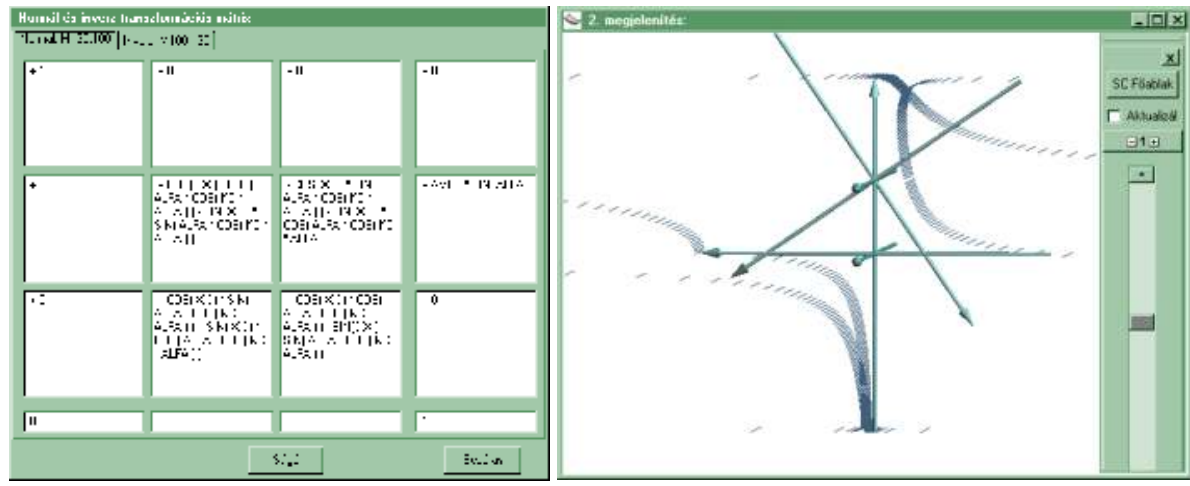

Fig. 19. The transformation matrix and the axoid of a complex motion

\section{Innovative solutions}

This section will present three innovations. The first one solves the problem of theoretically exact grinding of conical and globoid worms. This example demonstrates the calculation of a conjugate generated surface. The second solution applies double modification to the surface of a helical worm, providing smooth connection between the worm and the wormwheel. The third example presents the construction of a worm gearing having a point-like contact pattern.

\subsection{Theoretically exact grinding of non-helical worms}

This section introduces a special patented worm grinder machine construction and related technology that resolves the problem of geometrically exact grinding of conical and globoid worms. The new method proposed here applies a special grinding wheel having the same number of threads as the worm has. The novelty of this machine lies in the special grinding wheel that is not surface-of-revolution form, because the working surface of the wheel is generated as a conjugate surface pair of the worm.

The problem of the grinding of non-helical worms, which originates from the changing curvature values of tooth surface and changing diameter along the axis, was discovered decades ago, see (Boecker \& Rochel, 1964). Globoid worms - for example Cone-types - and spiroid worms are characterised by threads having different diameters. 
This property leads to changing curvature values along the threads of the worm, so application of the classical grinding wheel with surface of revolution form is impossible, because the wheel is not able to change its shape during grinding. However, this ability is required for the grinding of globoid or spiroid worms because the contact line between the machined worm and grinding wheel changes its shape over time. For the solution of the problem different methods are known. One of the suggested patented technologies applies a $\mathrm{CNC}$ grinding machine and $\mathrm{CNC}$ wheel dresser combination that continuously dresses the wheel during grinding (Dudás, 2000). It is possible to eliminate geometrical errors and to produce theoretically exact spiroid worms using an involute surface. This type of worms were developed and introduced in (Gansin, 1969; Tajnafói, 1966). There is a special generating method to produce globoid worms having possibility for exact grinding. In this case, the generating surface for globoid worm and for the gear is a surface of revolution (Siposs, 1992). The author of this chapter also proposed special grindable Archimedean and convolute spiroid worm constructions featuring a cylindrical foot surface, shown in Figure 20. If we would like to grind general spiroid or globoid worms in a theoretically exact manner, we can apply a new method that will be introduced in the following sections.

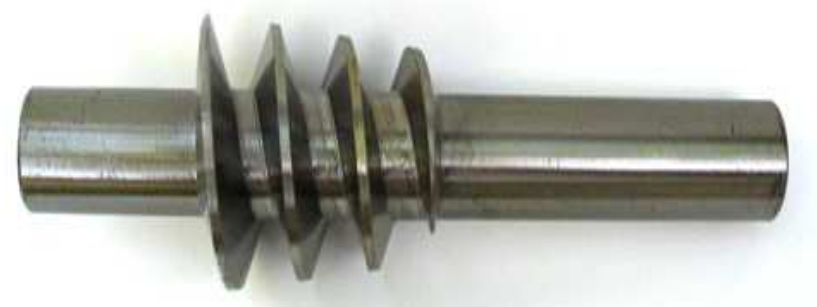

Fig. 20. A grinded Archimedean spiroid worm with cylindrical foot surface

\section{New Procedure and Grinding Machine Construction}

If the surface of revolution shaped wheel cannot change its shape, then exact machining needs a special grinding wheel that has different profiles for every diameter of the worm. A grinding wheel generated by the worm as its conjugate surface fulfils this requirement. The invention of the author (Dudás, 1990) includes such a wheel making the grinding of general spiroid or globoid worms possible in a theoretically exact manner, see Figure 21. The process applies the same angle speed quotient between the wheel and the worm for calculation of wheel surface. This angle speed quotient is generally equal to 1 and means opposite tangential speed directions (identical angle speed vectors). The form of the new type of grinding wheel may remind one of a Reishauer-type wheel or can take another form, like a toroid gearing-element. However, there is an important difference between the Reishauer-type wheel and this: the proposed wheel almost never has a helical working surface. The main advantage of the suggested construction and grinding process is that it makes theoretically exact grinding of globoid and spiroid worms possible. The disadvantage is the complicated manufacturing of the grinding wheel and the complex NC grinding machine. This type of grinding machine is advantageous for the mass production of worm surfaces, as opposed to continuous dressing during grinding, which is useful for the production of tool surfaces and smaller series of gearing elements. For a full explanation see (Dudas, 2007). 

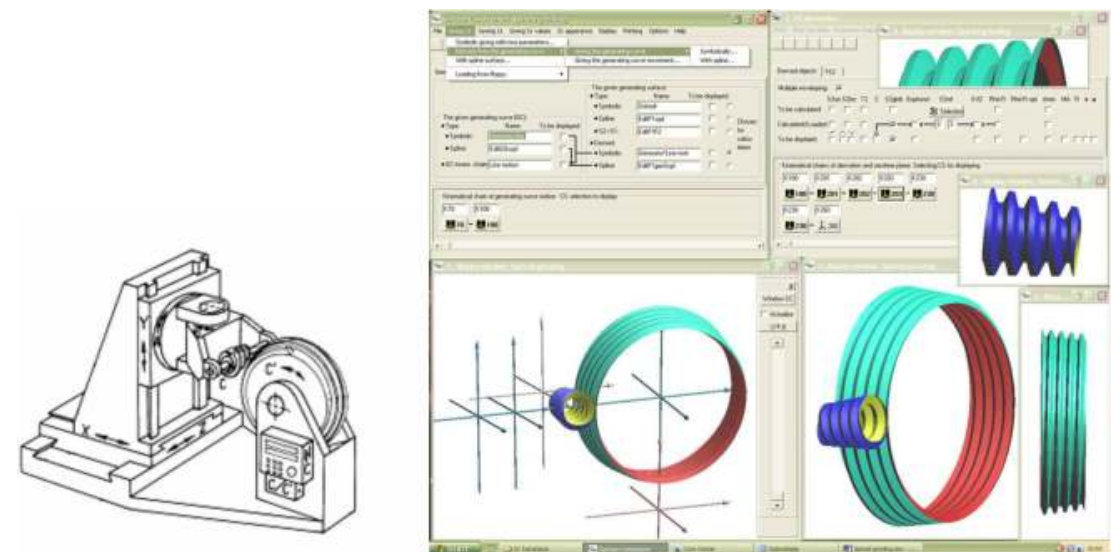

Fig. 21. The grinding machine and the grinding wheel generation by SC

\subsection{Modelling a double-modified worm gearing}

The next example will introduce the use of SC for investigating a new modification method for worm gearing with an elliptical generating curve in the axle plane of the worm.

The modification of gearing members is usually applied to achieve smooth starting and finishing of connection when the teeth enter or exit from the meshing, see (Litvin et al., 2001; Ohsima \& Yoshino, 2001; Seol, 2000). Though these transitions are smoother in the case of helical gearings than with the connection of spur gears, the effect of elastic deformation because of the load or manufacturing and assembly errors causes deviance from the designed exact conditions. The goal of the modification is to achieve a point-like contact pattern instead of the exact line form of connections having one motion parameter. A perfect modification method uses minimal surface modifying to preserve the quasi line connection pattern in the loaded state of gearing to make possible a long cuneal oil-yawn, and takes into account the tolerances specified for the manufacture of gearing parts and for assembly. The possibilities of worm gearing modifications are as follows:

- gear tooth profile modification using a slightly different hob for toothing than the hob that produces the conjugate gear

- gear tooth profile modification applying a different hob position at hobbing than the theoretically exact position

- $\quad$ worm tooth profile modification in the root and tip regions

- worm profile position modification resulting in a barrel-form worm instead of a cylindrical worm

- $\quad$ worm profile position modification along the worm length using changing pitch

- combinations of the above.

In the next section, the effect of pitch modification along the worm length will be analysed through the contact pattern for an elliptical worm profile in the axle plane. Then a second profile modification will be added to localize the connection to the central region of the working area of the teeth. Worm gearings with a circular profile in the axle plane of the worm have better connection properties than gearings with a linear profile, because contacting curves are closer to the radial direction and more perpendicular to the relative sliding speed direction. 
Applying the ellipse profile curve in the analysis of the connection characteristics gives one more degree of freedom and may result in better contact line shapes. The generating ellipse arc of the worm is determined in the K70 co-ordinate system (CS) in Figure 22. It is important to emphasize that the sketch of the profile and the relations of the CSs are intended for documentation purposes; the SC system visualises the entered relations and the investigation itself needs no such drawings. After opening a new project in SC, the generating curve can be entered symbolically, as on the right side of Figure 22.
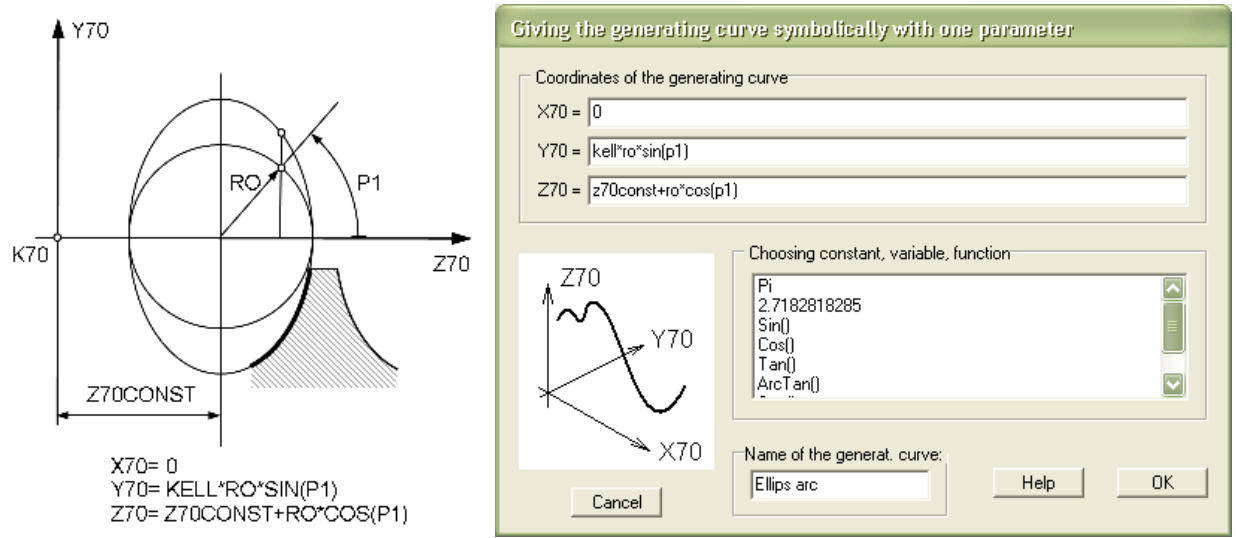

Fig. 22. The elliptic generating arc and entering it in SC
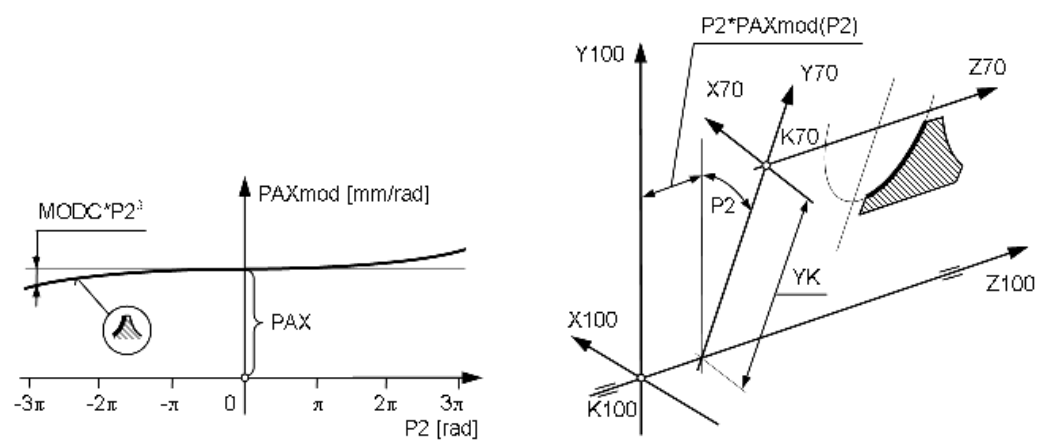

Fig. 23. To the left: Cubic function of P2 parameter for the left side of the worm tooth To the right: The use of cubic function for worm surface generation

The goal of the use of pitch modification is to allow a small gap for entering and exiting teeth in an unloaded condition that will decrease parallel to the increase of the load. This effect can be realised using an axial pitch modification along the worm length, as shown in Figure 23. The cubic function preserves the original conditions of the connection for the teeth of the worm in the middle section and results in a slightly larger gap to avoid interference with the entering and exiting exterior threads of the worm. In the function, $P 2$ is the generating parameter of the worm surface that is applied in the quasi-helical motion, as shown in the right side of Figure 23. Changing the sign of the MODC constant gives the modification function for the right side of the tooth. The K100 CS holds the worm. 


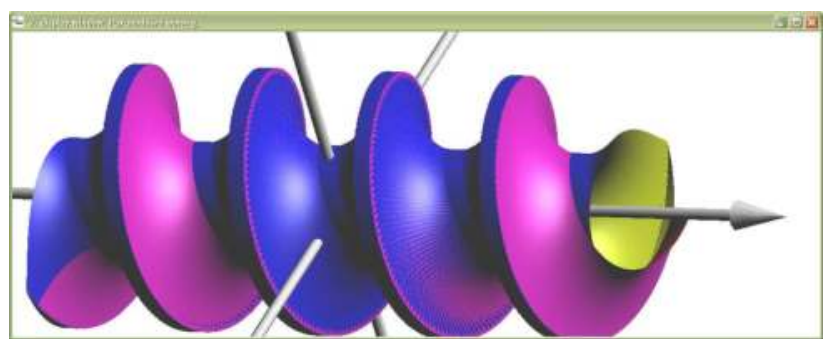

Fig. 24. The worm with axially modified tooth surfaces and the lighter reference helicoid

The creation of the co-ordinate transformation matrices and the symbolic multiplication of the concatenated matrices are accomplished automatically. After the values have been entered the objects defined on the symbolic level can be displayed. In this case some of the introduced steps had to be repeated to enter the right side of the tooth and the root and tip surfaces because of the different axial pitch functions of the two sides. Figure 24 displays the worm. To make visible the very small axial modification, the non-modified helical surface is also drawn using a different, lighter colour. This surface stands out from the modified surface, showing that the modification results in larger differences at the two ends of the worm.

The next step is to enter the kinematical relations between the worm and the worm-wheel, employing the sketch given in Figure 25. The K201 CS is fixed to the gearbox casing, while K230 holds the worm-wheel. The Z100 and Z230 axles are perpendicular.

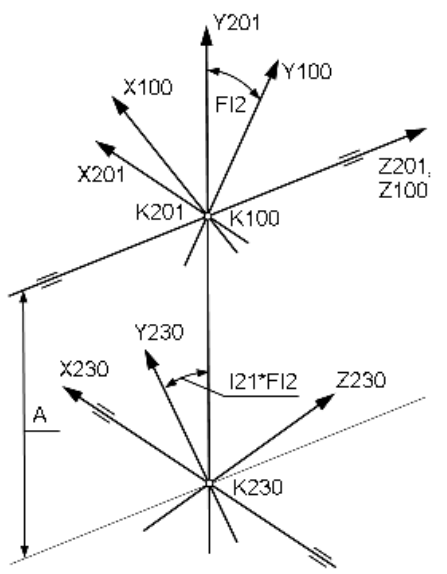

Fig. 25. Relation of the worm (K100) and worm-wheel (K230) CSs

Before starting the worm-wheel generating process, the ZETA2-dependent position of the RHO2-TAU2 plane is needed, where RHO2 is the reaching direction while TAU2 is the division co-ordinate direction. The RHO-TAU CS is limited to the plane CS in SC to achieve higher calculation speed, and can be any special curved CS, for example a polar system, but now this will be a simple planar Descartes system as in Figure 26. This slicing plane is assigned to K260 CS and will be positioned relatively to the worm-wheel by the BETA angle 
constant to consider the helix angle and by the ZETA2 slicing CS identifier parameter to intersect the surface of the wheel (see the right side of Figure 26). For every ZETA2 position an intersection curve of the worm-wheel will be determined by points that arose at different TAU2 values and are characterised by the calculated $\mathrm{RHO} 2$ value, as the extremum of the RHO2 values in the RHO2-FI2 co-ordinate surface, as was introduced generally in Figure 3.
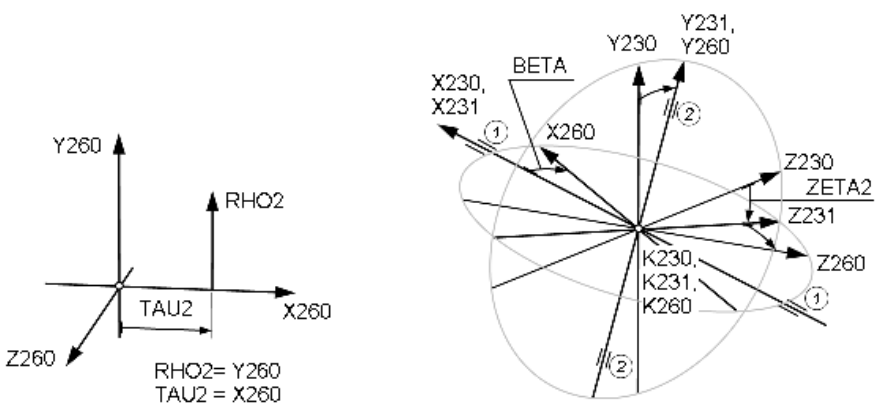

Fig. 26. Determining the $\mathrm{RHO} 2$ reaching direction in the X260-Y260 co-ordinate plane and positioning the slicing plane by ZETA2 CS identifying parameter and by BETA constant

The entering of these relations was accomplished similarly to the entering of the previously mentioned CS relations. After setting the reaching extremum to the global minimum, and entering the values for scalar and interval variables, the generating calculation can start. The resulting gear surface segment and the worm are presented in Figure 27.

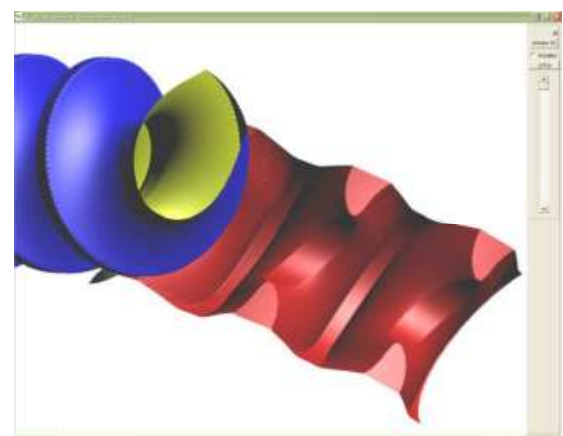

Fig. 27. The worm and the worm-wheel segment

Comparing the contact patterns of the modified and the unmodified worms

To check the effect of the pitch modification, the contact patterns of the modified and unmodified worms are analysed. In Figure 28 the modified version is shown on the left while the unmodified case is shown on the right. The worm is rolled around the fixed gear and the contact pattern is analysed from the inside of the gear. The wandering of the contact pattern on one side of the middle tooth is analysed. In case of non-modified, one-parametric enveloping, the points of the contact pattern are characterised by the same $F i$ value in the SC. It is possible to visualise any individual contact pattern as a set of points (Dudás, 1992). Visualisation in the following figures applies another method: it exploits the limited 
exactness of OpenGL visualisation in case of surfaces given in points of a two-parametric grid. The inner points of a grid segment are not in exact spatial position, and the generation of the surfaces by discrete motion simulation also causes errors, so the two connecting surfaces interfere with each other. Because the colours are different, the small intersections of the surfaces form a 'contact pattern'. This pattern is informative, but depends on the parameters of the grid and the simulation. Theoretically the contact pattern is a line or a point among two rigid solids. In practice the elasticity of the real gear material results in patterns with width. The applied method gives a realistic pattern, because implicitly it models the effect of distance between the points of the two surfaces along the theoretical contacting line.

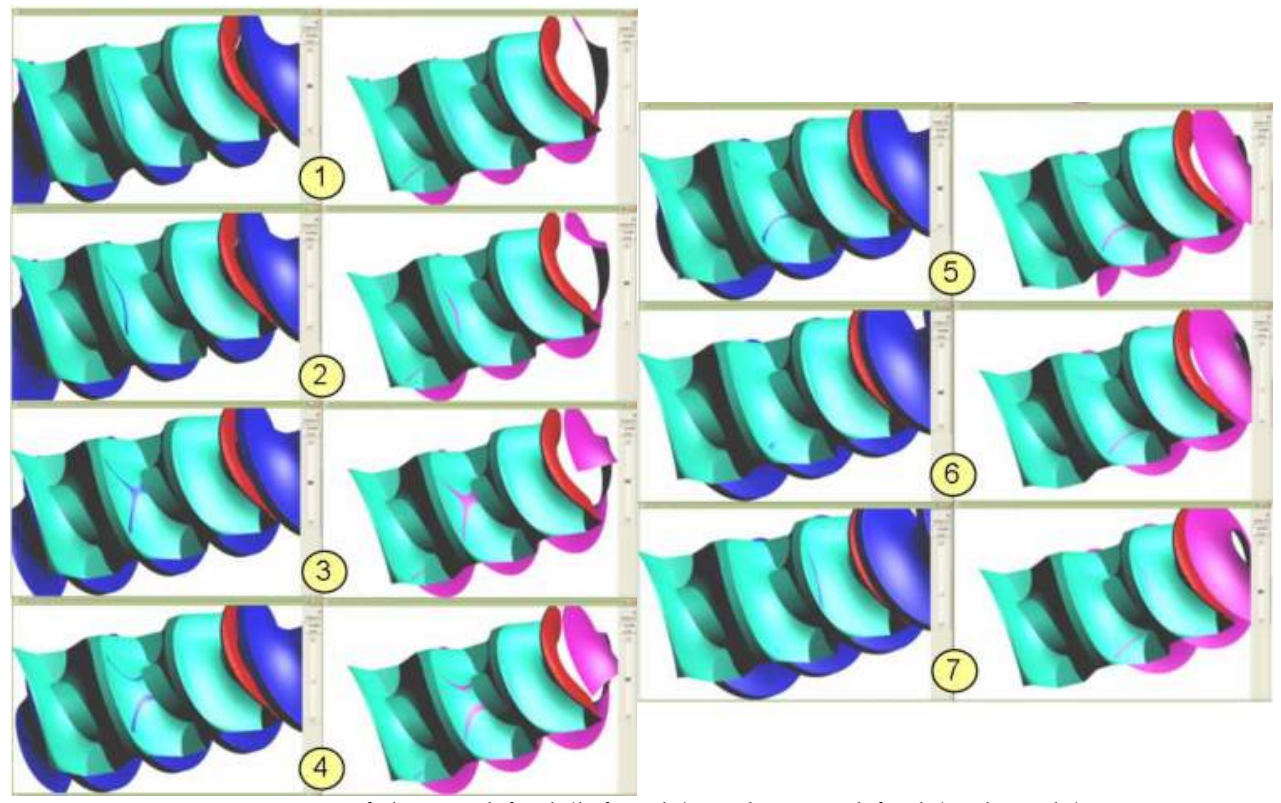

Fig. 28. Contact patterns of the modified (left side) and unmodified (right side) worms

When the worm enters the mesh the pattern has a tangential form, see Phase 1 in Figure 28. It is important to note that there is a radial contacting line on the previous tooth for the unmodified worm but there is no contact in the case of the modified worm. Considering that under load the middle tooth is elastically deformed, the connection can also develop on the left tooth. Depending on the value of the load, the value of the MODC modification parameter can be set properly in design time. Phase 2 is very similar. Phase 3 shows the moment when the contacting arcs, moving in the radial direction, meet. The tangential shapes change to radial, improving the contacting quality greatly. In the next phases the connection is characterised by advantageous radial patterns. In the Phase 6 , the right side tooth enters the mesh, and in the Phase 7 the middle tooth exits the mesh. 
Adding a second modification in radial direction

The comparison proved that the modified worm has localised to the middle of the worm connection. But the figures show that the patterns remaining after localisation have bits at the root and tip region too, which are very sensitive to misalignment and cannot tolerate assembly errors. To localize the connection area in the radial direction as well, the profile modification shown in Figure 29 can be used.

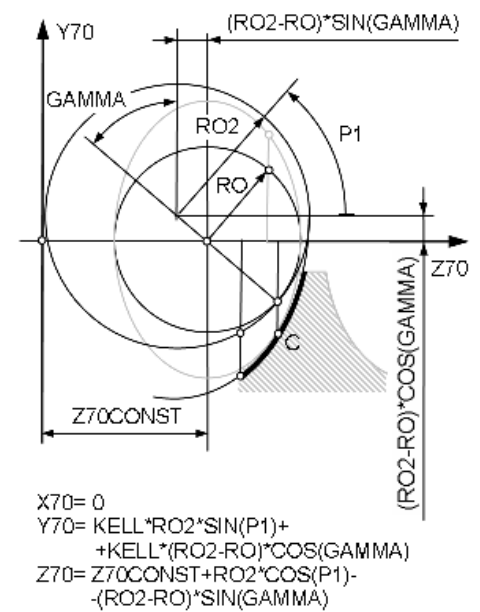

Fig. 29. Using a larger ellipse arc to modify the profile in radial direction of the worm

This new profile applies a larger ellipse arc and uses GAMMA angle to make it possible to set the position of the unmodified $C$ central point. By changing RO2 and GAMMA, the localisation can be fine tuned, as can be seen in Figure 30. To make the effect of this radial modification visible, the lighter modified surface was shifted by $0.005 \mathrm{~mm}$ relative to the worm surface with pitch modification only.

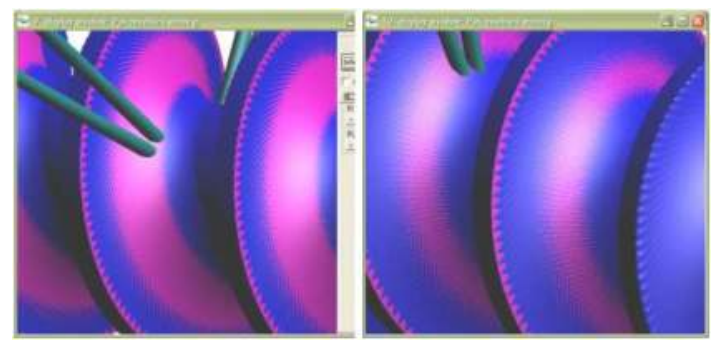

Fig. 30. Picturisation of different radial modifications on the worm: $R O 2-R O=0.2$ (left) and 0.05 (right), GAMMA= $47^{\circ}$

The contact patterns of the twice modified worm gearing are shown in Figure 31. The patterns are localised to the centre of the contacting area of the worm-wheel and the worm. 


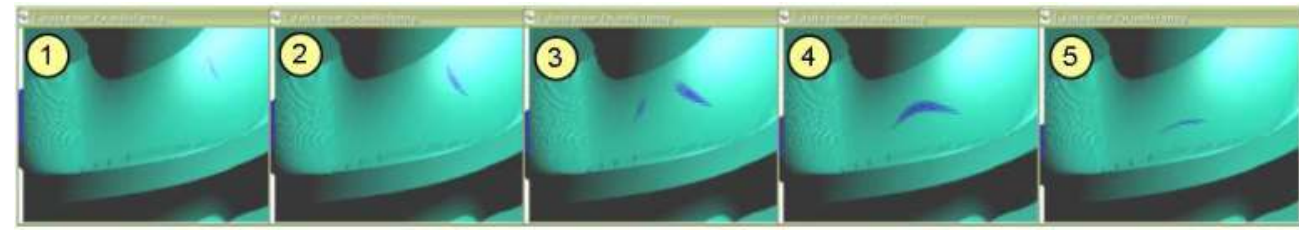

Fig. 31. Contact patterns of the twice modified worm gearing

The advantage of this twice-modified worm gearing is the localised contact and, as a consequence, smooth, noiseless working. The disadvantage is the problem of exact grinding that needs similar technique to that mentioned regarding conical and globoid worms. The problem was analysed in a detailed manner in (Dudás, 2008). Grinding with surface of revolution shape wheel causes $0.03-0.04 \mathrm{~mm}$ error. Like most of the modified gearings (Litvin \& De Donno, 1998), this worm gearing also has a very small alteration in the transfer ratio.

\subsection{Transmission error-free localised worm gear having point-like contact}

The SC gear modelling application is capable of producing gearings having point like contact. In Figure 8 the generated members of a gearing with a point-like connection are surfaces $F 2_{1}$ and $F 2_{2}$. The intermediary theoretical generating surface is $F 1$. The generating kinematical relations are given by transformation matrices as functions of Fi1 and Fi2 motion parameters. In the followings a theoretical helicoid with circle profile in the axle plane will be the intermediary generating surface.

The generating surface has to fulfil the following requirements:

- The contact curves appearing between $F 1$ and $F 2_{1}$ and between $F 1$ and $F 2_{2}$ must give an intersection point $M$ in every moment of the working of the worm gearing (on different teeth, providing a larger engagement factor than 1).

- The shape of the generating surface has to guarantee the machinability of the generated surfaces, the teeth of the worm and the worm-wheel.

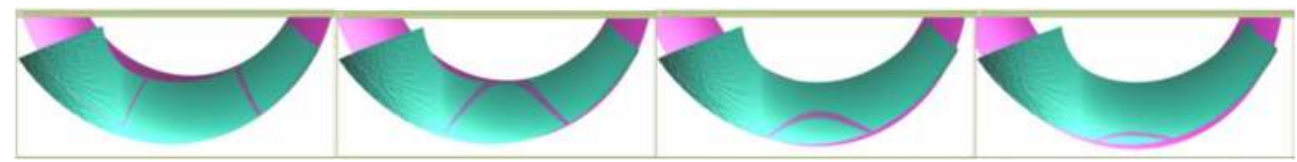

Fig. 32. The contact line of a ZTA-type worm gear is quasi-orthogonal to sliding speed

Guaranteeing a continuous point-like connection

The chosen $F 1$ generating helicoid will result in a constant $k 1$ contact curve between the $F 1$ and $F 2_{1}$ worm surface. We can predict that this will have a close-to-radial shape. On the other hand, the $k 2$ contact curve between the $F 1$ and $F 2_{2}$ worm-wheel surface will change by the minute. Figure 32 pictures the previous modelling of a ZTA-type worm gear connection. The forms of ZTA contact lines are advantageous for achieving a loadable oil film. The point-like connection will worsen these lines so the thickness of the oil film will not be as even along the "contact curve direction". Applying a slightly larger helicoid than the working tooth surface of the worm of a non-modified ZTA worm gearing will result in similar $k 2$ contact curves. So the chosen form of the $F 1$ generating surface will be a helicoid 


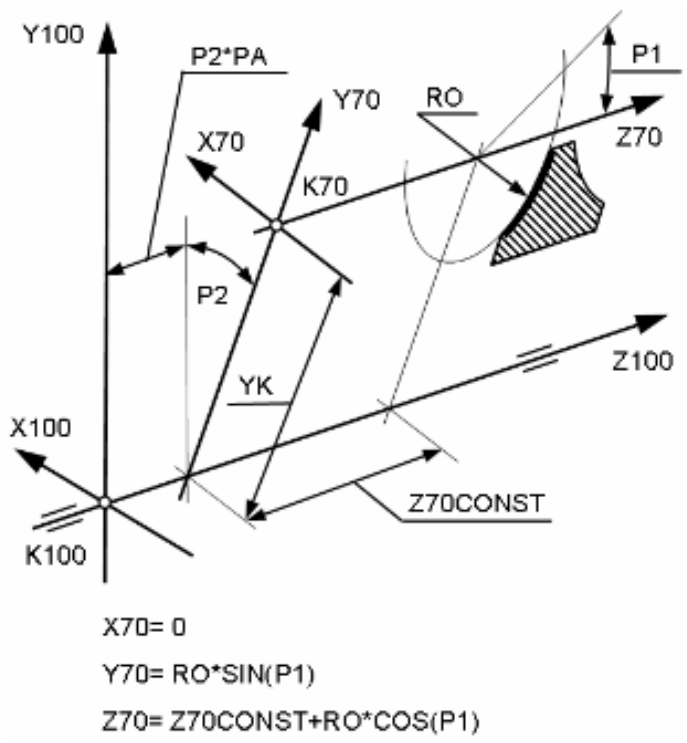

Fig. 33. Determining an $F 1$ intermediary generating helicoid as a surface swept by a circle-arc

having a circle arc in the axle plane, as in Figure 33. This helicoid will have a parallel axis to the generated $F 2_{1}$ worm surface, as shown in Figure 34. In addition to the DELTAY vertical distance that handles the difference between radiuses, a DELTAX horizontal displacement is introduced to make it possible to find a proper relative position for the F1 generating helicoid that will result in a quasi-radial $k 1$ contact line to create a continuous intersection with the $k 2$ contact curve of $F 1-F 2_{2}$ (see the right side of Figure 34).

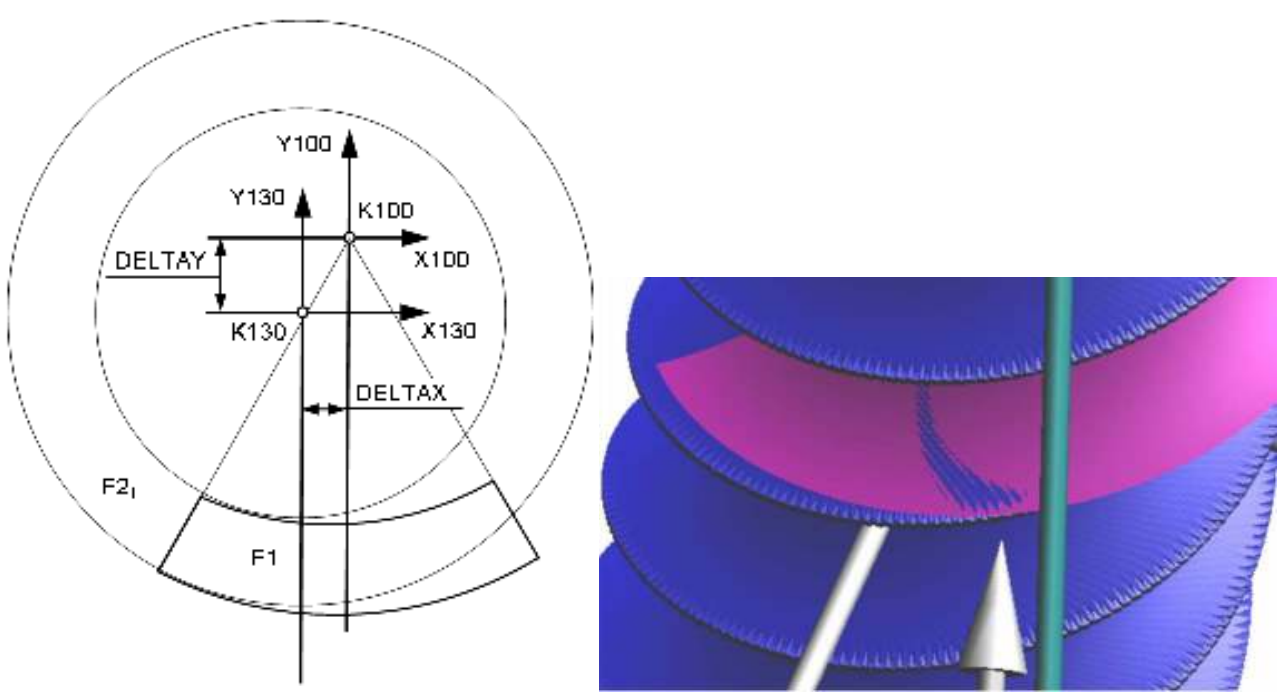

Fig. 34. Relative position of $F 1$ generating helicoid and $F 2_{1}$ generated worm surface 
For the practical generation in SC only a segment of the $F 1$ intermediary surface was applied, and instead of rotation of the generating helicoid the translation of the helicoid segment along its axis was applied exploiting the property that a helicoid can be twisted in itself. This speeded up the trial and error method which is used to determine suitable DELTAX and DELTAY values to ensure continuous intersection of contact curves. The circle arc profile generator curve was given in the $K 70$ frame, the $F 1$ generating helicoid segment was fixed to frame K100. The generating motion of helicoid segment F1 relative to the rotating $F 2_{1}$ generated worm can be analysed in Figure 35. The generated $F 2_{1}$ worm was calculated in the K130 frame using the K160 frame. This frame had an angle position in K130 determined by ZETA1 parameter (see Figure 36). The reaching direction RHO1 and divisioncoordinate TAU1 was defined in this K160 frame as shown on the right side of Figure 36.

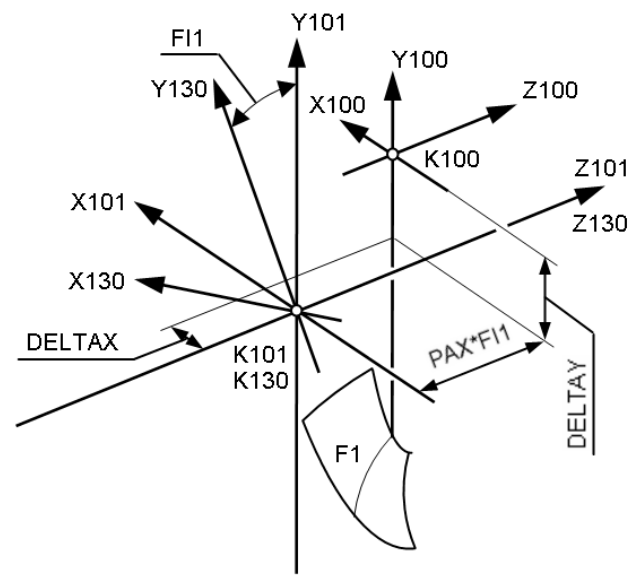

Fig. 35. The generating motion between K100 and K130 frames

Because the gearing has to work in the opposite rotational direction as well, the other side of the worm tooth requires the F1 surface to be rotated around Y101 axis by 180 degrees, as can be followed in Figure 37.
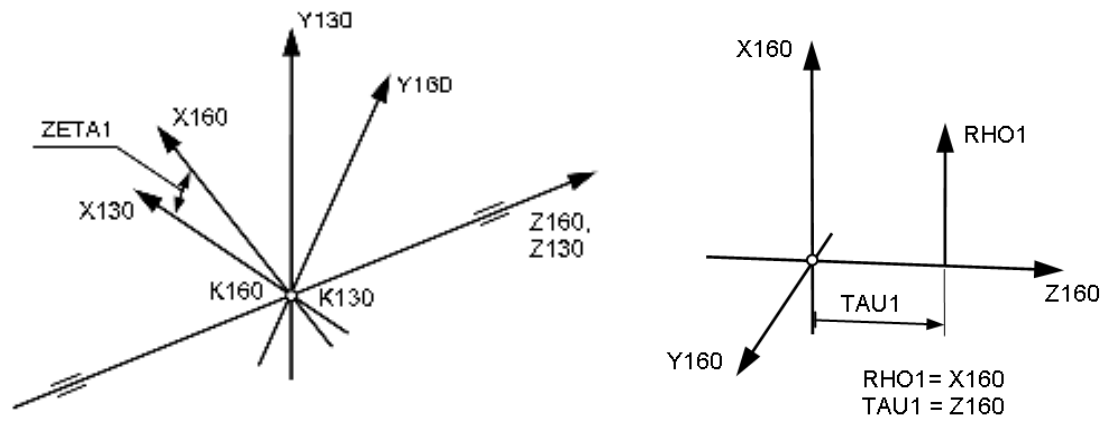

Fig. 36. Positioning the slicing plane $X 160-Z 160$ by ZETA1 angle parameter and determining the $R H O 1$ reaching direction in the X160-Z160 co-ordinate plane 


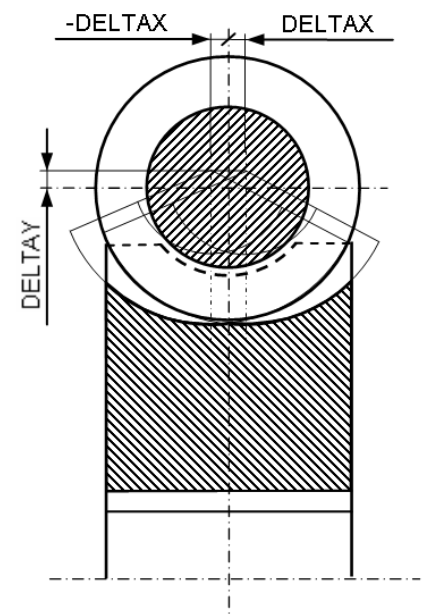

Fig. 37. The relation of the generating helicoid segments to the worm and worm gear

After determining the suitable $F 1$ surface and position settings that would guarantee the continuous intersection of $k 1$ and $k 2$ contact lines, the finishing steps were the generation of the missing tooth surfaces of the $F 2_{1}$ three-start worm and the generation of the missing surface parts of the $\mathrm{F}_{2}$ worm-wheel segment. The algebraic sign of DELTAX was different for the two sides of the teeth. For the calculations the global method was selected in SC for both generated gearing elements.

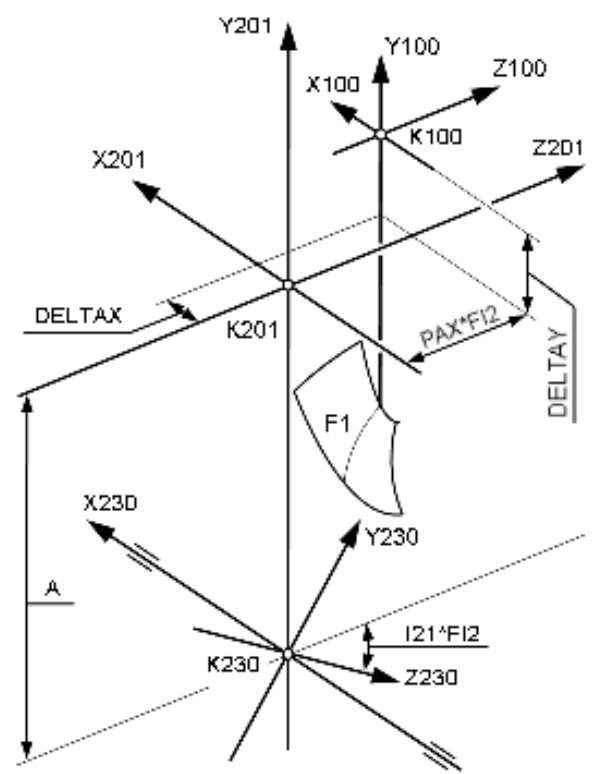

Fig. 38. Determining the relation of the generating helicoid segment (K100) and worm-wheel (K230) frames 
The kinematical arrangements applied in the generation of the $\mathrm{F}_{2}$ worm gear surface are described below. The kinematical relation between the $F 1$ generating helicoid segment and the generated gear tooth surface is sketched in Figure 39. F1 is fixed to the K100 frame and performs a linear motion determined by the Fi2 motion parameter while the calculated gear surface rotates around an X230 axis. Similarly to the generating process of the $F 2_{1}$ worm surface, the calculation of $\mathrm{F2}_{2}$ points required the determination of the ZETA2-dependant position of the RHO2-TAU2 plane. This relation is similar to solutions showed in Figure 26. Figure 39 shows the SC gearing design tool in the midst of development. The upper left window shows a triple start worm and the worm-wheel segment in the midst of motion simulation. Under it the intermediary F1 generating helicoid segment, the generated F21 worm and the constant $\mathrm{k} 1$ contact line can be checked. The upper right window shows the F1 generating helicoids segment and the generated $\mathrm{F}_{2}$ worm-wheel segment. The momentary contact curve forms an arc. The lower-left display window shows the generated worm, the generated worm-wheel and the intermediary generating helicoid segment. The point-like contact of the worm and worm-wheel appears as a darker spot on the contact arc of the generating surface and the worm-wheel. This spot can also be seen in the fifth window.
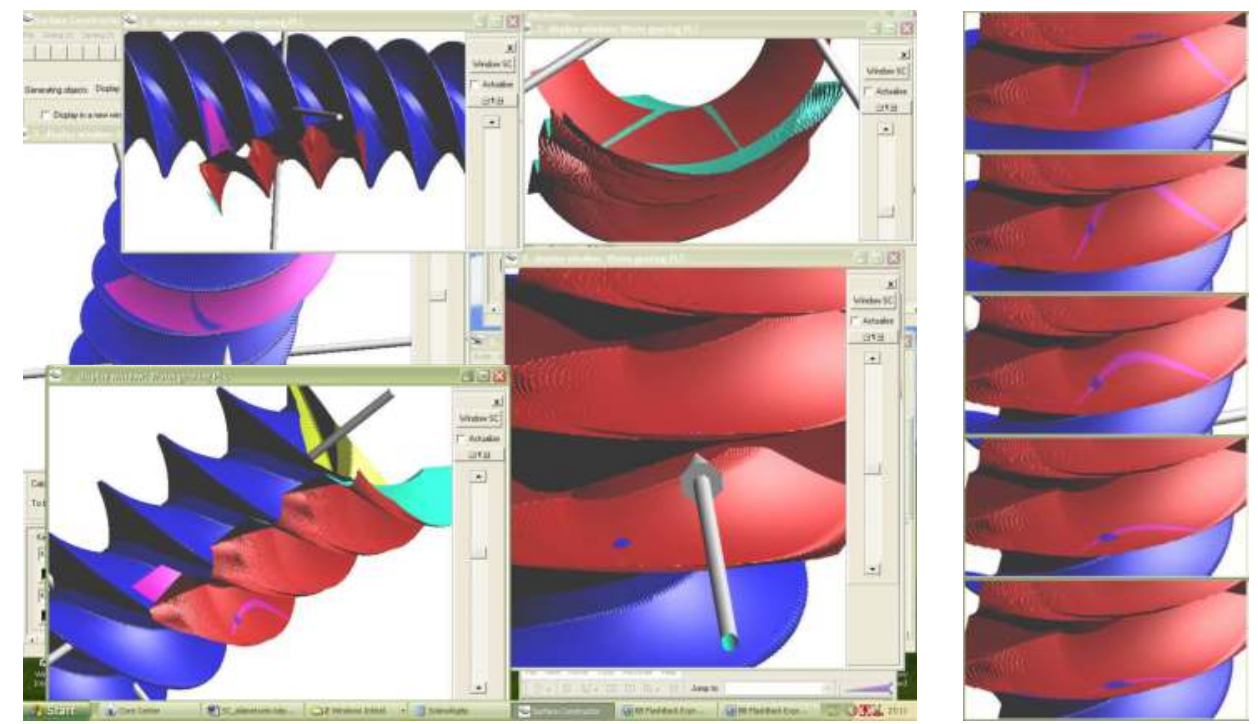

Fig. 39. To the left: The Surface Constructor kinematical modelling and simulation software visualises the gearing elements

To the right: Analysis of contact pattern in different moments of meshing. The darker 'patch' is the location of the point-like contact

The meshing analysis proved that the contact is continuous and the engagement factor never goes below one. This can be checked in Figure 39, to the right, in the uppermost subfigure, where contact patterns appear on two teeth simultaneously. The results of global cuts were detected and evaluated. While there is a large interference region on the tooth of the worm wheel, this does not affect the meshing.

The goal of the research documented here was to prove the viability of a new type of worm gearing that has localised contact pattern but works with zero transmission error. 


\section{Conclusion}

This chapter has reviewed some results achieved using the Surface Constructor gears connection modelling application in the area of gearings development. The importance of the Reaching Model theory as the foundation of the tool was discussed. Then a short description presented the structure of the software and some capabilities of it. The emphasised versatility originates from the symbolic algebraic calculation, which is one of the special features of the software. In the main part of the chapter, some solved tasks were shown. The first investigation gave a possible solution for the problem of grinding of the non-helical worms in a theoretically exact way. The second demonstration presented a double modified worm gearing having a localised bearing pattern. The last example was a method of producing gearings having point-like connection, which was demonstrated by the creation of a novel worm gearing type characterised by continuous meshing in a point, resulted from the use of an intermediary generating surface, thus avoiding transmission error. These examples prove the applicability of Surface Constructor for gear investigation and innovation.

\section{Acknowledgements}

This chapter was prepared within the framework of the TAMOP-4.2.1-08/1-2008-0006 project titled "Development and Operation of the Technology and Knowledge Transfer Center of the University of Miskolc". The project takes place with the support of the European Union, co-financed by the European Social Fund.

\section{References}

Boecker, E. \& Rochel, G. (1964). Messprobleme bei der Fertigung von snecken-getrieben. Werkstatt und Betrieb, No.2, pp.153-156

Dudás, I. (2000). The Theory and Practice of Worm Gear Drives, Penton Press, ISBN1857180275, London

Dudás, L. (1990). Grinding machine for grinding free form surfaces located on surfaces of revolution, especially for spiroid and globoid worms, Invention No. OTH19089/1990.06.12. Publishing No. H/3615, Hungarian Patent Office

Dudás, L. (1992). Resolution of Geometrical Problems of Contacting Surfaces Using the Reaching Model, (in Hungarian), PhD Thesis, Hungarian Academy of Sciences, Budapest

Dudás, L. (2007). Possibilities of Exact Grinding of Conical and Globoid Worms. International Review of Mechanical Engineering (I.R.E.M.E.), Vol. 1., No. 3., (May 2007) pp. 200-207, ISSN 1970-8734

Dudás, L. (2008). Developing a Universal Gearing Design and Gear Manufacturing Investigation Tool, Proceedings of TMCE 2008 Symposium, pp. 1231-1245, ISBN 9789051550443, Izmir, Turkye, April 2008, Delft University of Technology, Delft

Gansin, V. A. (1969). Grinding Worm of Involute Spiroid Gearings, Machine Tools and Tools, No.5., pp. 25-27. 
Kato, S. \& Kubo, A. (1999). Analysis of the effect of cutting dimensions on the performance of hypoid gears manufactured by the face hobbing process, Proceedings of the 4th World Congress on Gearing and Power Transmissions. pp.585-593, CNIT-PARIS, France.

Kissling, U. L. (1999). Noise and vibration reduction in cylindrical gears by an accurate procedure implemented in KISSsoft. Proceedings of the 4th World Congress on Gearing and Power Transmissions. CNIT-PARIS France. pp. 117-128.

Litvin, F. L. (1994). Gear Geometry and Applied Theory, Prentice Hall, ISBN0132110954 Englewood Cliffs, NJ.

Litvin, F. L. \& De Donno, M. (1998). Computerized Design and Generation of Modified Spiroid Worm-Gear Drive With Low Transmission Errors and Stabilized Bearing Contact, Computer Methods in Applied Mechanics and Engineering, Vol. 162, No. 1-4, pp. 187-201.

Litvin, F. L. \& Fuentes, A. (2004). Gear Geometry and Applied Theory, Cambridge University Press, ISBN0521815177, Cambridge.

Litvin, F. L.; Fuentes, A. ; Hawkins, J. M. \& Handschuh, R. F. (2001). Design, Generation and Tooth Contact Analysis (TCA) of Asymmetric Face Gear Drive With Modified Geometry, NASA-TM-2001-210614.

Lunin, S. (2010). Design of worm gears for low noise applications using worm gear software from ZAKGEAR, http:/ / www.zakgear.com/Worm.html , Accessed: 2010.05.20.

Miltenovic, V. \& Milcic, D. (1999). Intelligent integrated system for the gear power transmitters design. Proceedings of the 4th World Congress on Gearing and Power Transmissions, pp. 595-606. CNIT-PARIS, France.

Ohshima, F. \& Yoshino, H. (2001). Ideal Tooth Surface Modification Method For Cylindrical Worm Gears, Proceedings of the JSME International Conference on Motion and Power Transmissions, pp. 460-465, VT:MPT II(01-202).

Ramahi, A. (1998). On the Kinematics of Spatial Gearings UMTIK'98 International Machine Design and Production Conference, pp. 37-45, Ankara, Turkey, Sept.9-11.

Seol, I. (2000). The Design, Generation, and Simulation of Meshing of Worm-Gear Drive With Longitudinally Localized Contacts, Journal of Mechanical Design, Vol. 122, No. 2, pp. 201-206.

Seveleva, G. I.; Gundaev, S. A. \& Pogorelov, V. S. (1989). Numerical Modelling of Cutting Tapered Gears having Teeth with Circular Arc Form (in Russian), Vestnik Mashinostroenia, No. 3, pp. 44-47.

Siposs, I. (1992). Globoidschneckengetriebe mit Kreisbogenprofil, Publications of the University of Miskolc, Series C, Mechanical Engineering, Vol. 44, No. 1, pp. 7-19.

Stadtfeld, H. J. (1999). The Universal Motion Concept for Bevel Gear Production. Proceedings of the 4th World Congress on Gearing and Power Transmissions CNIT-PARIS France. pp. 595-606.

Su, D. \& Qin, D. (2003). Integration of Numerical Analysis, Virtual Simulation and Finite Element Analysis for the Optimum Design of Worm Gearing, Journal of Materials Processing Technology, Vol. 138, No. 1-3, pp. 429-435.

Tajnaföi, J. (1966). Theory and a Few Application of Motion Describing Capabilities of Machine Tools, Ph.D. Thesis, Dept. of Machine Tools (in Hungarian), Univ. of Heavy Industry, Miskolc. 


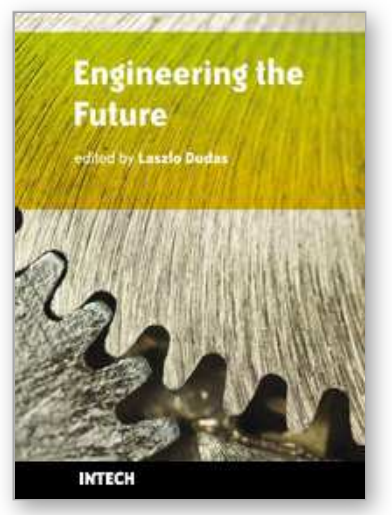

\author{
Engineering the Future \\ Edited by Laszlo Dudas
}

ISBN 978-953-307-210-4

Hard cover, 414 pages

Publisher Sciyo

Published online 02, November, 2010

Published in print edition November, 2010

This book pilots the reader into the future. The first three chapters introduce new materials and material processing methods. Then five chapters present innovative new design directions and solutions. The main section of the book contains ten chapters organized around problems and methods of manufacturing and technology, from cutting process optimisation through maintenance and control to the Digital Factory. The last two chapters deal with information and energy, as the foundations of a prospering economy.

\title{
How to reference
}

In order to correctly reference this scholarly work, feel free to copy and paste the following:

Laszlo Dudas (2010). New Way for the Innovation of Gear Types, Engineering the Future, Laszlo Dudas (Ed.), ISBN: 978-953-307-210-4, InTech, Available from: http://www.intechopen.com/books/engineering-thefuture/new-way-for-the-innovation-of-gear-types

\section{INTECH}

open science | open minds

\author{
InTech Europe \\ University Campus STeP Ri \\ Slavka Krautzeka 83/A \\ 51000 Rijeka, Croatia \\ Phone: +385 (51) 770447 \\ Fax: +385 (51) 686166 \\ www.intechopen.com
}

\author{
InTech China \\ Unit 405, Office Block, Hotel Equatorial Shanghai \\ No.65, Yan An Road (West), Shanghai, 200040, China \\ 中国上海市延安西路65号上海国际贵都大饭店办公楼405单元 \\ Phone: +86-21-62489820 \\ Fax: +86-21-62489821
}


(C) 2010 The Author(s). Licensee IntechOpen. This chapter is distributed under the terms of the Creative Commons Attribution-NonCommercialShareAlike-3.0 License, which permits use, distribution and reproduction for non-commercial purposes, provided the original is properly cited and derivative works building on this content are distributed under the same license. 